\title{
The Evolution of Technology and the Mathematics of Change and Variation: Using Human Perceptions and Emotions to Make Sense of Powerful Ideas
}

\author{
David Tall
}

Institute for Education

University of Warwick

Coventry CV4 7AL, UK

david.tall@warwick.ac.uk

\begin{abstract}
This chapter reflects on the evolution of the mathematics of change and variation as technology affords the possibility of conceptualising and communicating ideas for a wider range of learners than the few who traditionally study the higher levels of the calculus. It considers the overall program of development conceived by Jim Kaput, instantiated in the software SimCalc as part of a full range of development using technology for 'expressing, communicating, reasoning, computing, abstracting, generalising, and formalising mathematical ideas.' The development begins with interactive representations of dynamic real world situations and extends the perceptual ideas of continuity and linearity through the operational symbolism of the calculus and on to the formalising power of mathematical analysis. It reveals that the Kaput program has the distinction that its overall framework contains the essence for continuing the complementary evolution of technology and the conceptions of mathematical change and variation. Furthermore, it envisages changes that we have, as yet, not implemented, such is the speed of technological change. In particular, new technology enables us not only to build more powerful ways of performing numerical and symbolic algorithms that may be represented visually and dynamically, it also provides new forms of input and gesture to offer an embodied, kinesthetic, and emotionally powerful experience of engaging with mathematics. This can be shared widely through fundamental human perception and action and can develop in the longer term through symbolism and human reason to the mathematical literacy required of today's citizens, the theoretical applications of mathematics essential for today's society and on to the boundaries of mathematical research that takes us into the future.
\end{abstract}




\title{
1. The Changing Nature of Technology
}

\author{
Anyone who presumes to describe the roles of technology in mathematics education faces \\ challenges akin to describing a newly active volcano - the mathematical mountain is \\ changing before our eyes ... (Kaput, 1992, p. 515)
}

This quote from Jim Kaput, written two decades ago, is just as apt today as it was then. As I began preparing this chapter, my iPad rang and my three-year old grandson came on line, demanding to speak to Tufty, our cat. Modern technology provides us with astounding ways of operating and communicating that were unimaginable not long ago. Yet while three-year-old children are embracing technology in a natural way, educators are having great difficulty in coming to terms with how to use it in teaching and learning.

The difficulty is not hard to diagnose. The speed of change of technology is so much faster than the possibilities of curriculum change which, in turn, must take account of the rate of cultural change. Thus, while a child may pick up an iPad, with software carefully designed for ease of use, and discover ways to use it for personal benefit, the curriculum designer must take time to reflect deeply on the complex issues that arise in our society and change over the longer term. Kaput succinctly formulated his own version of the situation in the following quote from near the beginning of this book where I have added italics to highlight important aspects that will be reflected upon in this chapter.

While our universe of experience can be apprehended and organized in many waysthrough the arts, the humanities, the physical and social sciences - important aspects of our experience can be approached through systematic study of patterns. In addition, mathematics embodies languages for expressing, communicating, reasoning, computing, abstracting, generalising, and formalising - all extending the limited powers of the human mind. Finally, mathematics embodies systematic forms of reasoning and argument to help establish the certainty, generality, and reliability of our mathematical assertions. We take as a starting point that all of these aspects of mathematics change over time, and that they are especially sensitive to the media and representation systems in which they are instantiated. (Kaput \& Roschelle, 2012, this volume, p. xxx)

Having written a calculus text (Fleming \& Kaput, 1979), Kaput went on to rail against the standard approaches to algebra and calculus that had the effect of steadily reducing the number of individuals that make sense of the subject, seeking instead a natural way to allow the wider population to gain the insight necessary for them to function as citizens with full democratic rights and access to knowledge. He saw this through the full development of 'expressing, communicating, reasoning, computing, abstracting, generalising and formalising' but he also realized that these aspects change and 'are especially sensitive to the media and representations in which they are instantiated.'

This applies not only to the technology that we use and develop, but also to our own personal development based on our previous experiences in life that may support or hinder our grasp of new ideas. I will illustrate this fundamental issue by 
recalling a difference of vision that occurred between Jim Kaput and myself that I now see in terms of his own insightful vision of the changing nature of technology as 'the mathematical mountain is changing before our eyes.'

\section{A Challenging Difference and a Resolution Using Technology}

Over the years Jim Kaput and I met in various parts of the world to share ideas. Though our goals in building from personal experiences to increasingly sophisticated ideas were broadly consistent, our own personal developments caused us to focus on different aspects. His experience with the 'big three' representations using expressions, graphs, and tables saw him focusing on ways of making links between them using technology.

He found the particular technology of 'pointing and clicking' a mouse could quickly draw a curve graphically to represent a real life story to give a new foundation for these fundamental ideas of the calculus prior to the use of expressions or tables. It encourages a much more general notion of function than is possible in traditional calculus, which essentially focuses on the symbolic manipulation of regular expressions using the 'rules of calculus' to derive the rate of change or to integrate to find the growth of a changing quantity.

My concern was more elemental. I wanted to 'see' and 'feel' change in a human sense through drawing a graph by the dynamic continuous movement of a finger or the use of a pencil.

We also differed in the extent of our vision. Jim focused on the wider democratic and social issues and had no desire to follow through to the formal development of traditional mathematical analysis, which he saw as the province of a privileged few. I wished to understand the full journey through the human development of mathematics itself, from the early experiences of the child to its eventual formalization and on to the frontiers of mathematical research. I also wished to develop a framework that predicted and explained why students followed such different paths of development in mathematics where the traditional curriculum seemed to steadily deny access to more and more learners.

Jim's use of 'pointing and clicking' gave him a notion of 'piecewise linear' graphs which could be described precisely, based on his own formal experience of mathematics, such as calculating a piecewise linear approximation to the area under a curve. My own very different experience, included teaching non-standard analysis to undergraduates who had already met standard analysis, where I could prove a formal theorem that 'a differentiable function', when 'locally magnified by an infinite scaling factor', would 'look like a straight line' (Tall, 1981, 2009).

Both of us were imprisoned in our own cultural experiences, which proved to be obstacles in our attempts to communicate our ideas to each other. I saw his piecewise linear functions as a fine mathematical idea that was well-known to mathematicians and worked well for calculating areas. Yet, for me, it had the flaw 
in calculating derivatives that the graph of its rate of change consisted of discrete horizontal line segments (Fig, xxx, p. xxx). Thus his vision took natural 'continuous' change and represented its 'rate of change' in a form that is certainly not 'continuous' in any intuitive human sense.

Meanwhile my idea of 'infinite magnification' of an 'infinitesimal' part of a graph flew against current trends in mathematical analysis where infinitesimals were seen to be an aberration of the past that had been replaced by the inscrutable but mathematically sound notion of the epsilon-delta definition of a limit. Even though I produced software that allowed the user to magnify graphs on the screen to see what is termed 'local straightness', my insights were seen as an interesting starting point to a calculus industry that remained wedded to its traditional development based on an 'intuitive' version of the formal limit concept with technology 'added on.'

On reflection, I can now see how both our visions may be explained in terms of his general theoretical perspective and how the changing technology has affected conceptions that are especially sensitive to the media and representations in which they are instantiated.'

In chapter xxx (Burke et al. 2012, this book), the long history of SimCalc has shown how theoretical ideas have to be adapted to fit with the changing technology, just as the technology changes to fit new ideas. Theoretical frameworks and technological innovations co-evolve (Hegedus \& Moreno-Armello, 2012, this book).

The iPad, which appeared only in 2010, was initially misunderstood with derisory comments from the cognoscenti such as Bill Gates who declared 'It's a nice reader, but there's nothing on the iPad I look at and say, "Oh, I wish Microsoft had done it."' (Gates, 2010).

Now in its third iteration, the iPad boasts a 'retinal display' so that what one sees on the screen held at a comfortable distance is at the maximum level of accuracy that can be seen by the receptors on the human retina.

The iPad also offers radical new modes of operation. One of these is to draw a graph with a movement of a finger, another is to control the display of an already drawn graph (which has an appropriate method of computation, such as a function formula) to picture it at any desired scale.

The drawing of a graph with a finger on the current iPad lacks precision, although it might be possible to draw a graph more accurately with a yet-to-be designed combination of enactive finger pointing and more precise action using a pen or mouse. This idea, favored by Bill Gates, exists on Wacom Bamboo tablets and is beginning to appear on touch screens. But even here, the precision of drawing is limited to that of a retinal display and graphs need to be imagined as being suitably smooth for it to have a continuously changing derivative.

Adobe Illustrator, using a mouse or tablet interface on a computer, already has the facility for drawing a graph with a pencil and then to select another tool to smooth parts of the graph (Figure 1). 


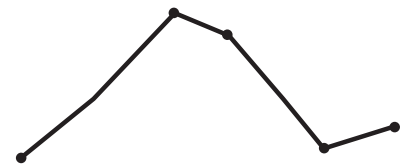

(i): Point and click

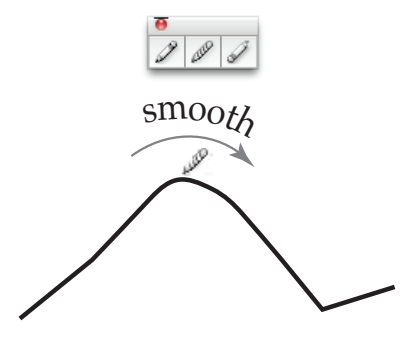

(ii): Smooth

Figure 1. Smoothing a graph.

I can see Jim in my imagination now, as he would turn up at a conference in the $80 \mathrm{~s}$ and $90 \mathrm{~s}$ to show the latest software, such as Excel in its earlier incarnations, that allowed him to imagine linking together symbolism and visualization in creative ways. Only now he might be looking at the iPad, drawing not with a point and click mouse, but with a finger, or with a more accurate pen, and then smooth out the graph he had drawn. He might also organize his input to touch specific points on the graph to type their actual values, or touch a part of the curve between specific points to input a formula. He might smooth the graph as in Illustrator, so that it became locally straight, or if he wished, he could use techniques already existing in Illustrator to draw a corner with different left and right tangents.

Such software would enable the learner to use a finger or pointer to draw a suitably accurate representation of a suitably smooth graph, and to 'crystallize' it (in the sense of Moreno-Armella \& Hegedus, 2012, in this book) from a dynamic movement into a static picture where now its rate of change could again be dynamically continuous.

Visually a differentiable function is 'locally straight' in the sense that, if the graph through a point where the function is differentiable is magnified, it will successively look less and less curved until, under high magnification, its graph looks like a straight line (Tall, 1985). We already have multi-touch technology such as the iPad where the user can touch the screen with finger and thumb and move them apart to cause the screen to be magnified. If this is programmed to keep the horizontal and vertical scales the same, then the slope of the curve can be seen under high magnification as the slope of a highly magnified segment that is visually a straight line. If two windows are available, one to show the graph and another to show the magnified part of the graph, it is possible to trace the finger along the graph and see the changing slope of the magnified part.

On the other hand, if the program offers a separate facility to stretch the graph horizontally and not vertically, then a continuous graph will 'pull flat' as I have long advocated (Tall, 1986, 2009, 2012). In this way, continuity and differentiability have simple interpretations in terms of different kinds of change of scale. 


\section{The Evolution of Ideas Using Technology}

This single example of different views of a complex issue illustrates a profound fundamental aspect of the evolution of ideas. As mere mortals we can only focus on a small number of factors and the differing ways in which we do so affect, and are affected by, the development of technology. It is not enough just to reflect on the nature of the changing technology or on the nature of mathematics as we see it at the time and on children's growing conceptions and misconceptions. We need also be confident enough to reflect on the validity of our own ideas as our cultures evolve and technology changes.

In an earlier chapter, Harel has characterized Kaput's work on the mathematics of change and variation as follows:

In all, Kaput's work on the mathematics of change and variation may be viewed as a research program - a program for which Kaput paved the foundations and offered a path for progress. Such a program can be characterized as one that pays a serious attention to: equity, quantitative meaning, gradual development (from elementary school onward), advanced-technology-based curriculum that is grounded in classroom context, and consistent epistemology. (Harel 2012, this volume, p. $\mathrm{xxx}$ )

This formulates what he terms 'the Kaputian program' as a broader research enterprise focusing on the mathematics of change and variation. It must be taken in conjunction with Kaput's ideas expressed earlier that seek to address the whole framework of building powerful mathematical ideas developing from the child's personal experience through modes of 'expressing, communicating, reasoning, computing, abstracting, generalising, and formalizing - all extending the limited powers of the human mind.'

Kaput's reference to the limited powers of the human mind applies not just to the children we teach or to the politicians who set the legal agenda for the mathematics curriculum, they also apply to us 'experts' and to Kaput himself. The amazing feature of his program is that it contains within it a vision that foresees the need to modify and evolve our own theories, even his own.

His program operates at two levels: the specific level of how we plan and deliver the curriculum using technological facilities such as SimCalc and the metalevel in which we constantly refresh and evolve the ways in which we think about how individuals make sense of mathematics.

Kaput's theory is a grand design, much bigger than the specifics of SimCalc. Though the implementation of SimCalc is vast in terms of the number of research studies that have been devoted to it and it focuses on issues beyond the mathematical content alone, in practice it has so far concentrated on a new vision of school algebra and the early development of mathematical variability and change.

Kaput crucially extends his vision of mathematics in school saying 'finally, mathematics embodies systematic forms of reasoning and argument to help establish the certainty, generality, and reliability of our mathematical assertions.' This potent vision extends his program through to the frontiers of mathematical thinking at the highest levels of mathematical research. 


\section{Extending the Kaputian Program}

In the 1990s, the Advanced Mathematical Thinking group of PME worked together to extend mathematics education to the formal mathematics experienced at university in which Harel \& Kaput (1991) extended ideas to more formal aspects of functions and calculus. In particular, they distinguished between the pointwise, local and global aspects of the calculus. Formal mathematics focuses initially on the limit concept at a specific point involving local behavior near that point and then extends definitions of continuity and differentiability by varying that point over the whole domain, leading to further formal distinctions between pointwise continuity and uniform continuity over an interval.

An embodied approach works locally and dynamically, shifting a finger over an interval in time and space as the moving finger leaves a trace of the underlying variation. It does not build from a technical definition of continuity at a single point to then apply this pointwise definition to every individual point over an interval. Dynamic continuity is a single gestalt, shifting attention along an interval as dynamically changing quantities vary together. It is peculiarly well-suited to the use of dynamic interactive technology.

Reflecting on many aspects of learning over many years with the help of colleagues and students has led me to extend childhood experiences of perception and action to ideas of advanced mathematical thinking as used at a more formal level (Tall, et al., 2001; Tall, 2006). This builds on human perceptions and actions and their consequences in terms of symbolism and proof. It is based on the conceptual embodiment of our perception and action where our actions - such as counting, measuring, adding, subtracting, evaluating, differentiating, integrating-may be symbolised and compressed into operational symbolism and then formalised in various ways. Formal thinking is expressed in terms of definitions and deductions.

However, as Kaput says insightfully, 'mathematics embodies systematic forms of reasoning and argument' and the forms of reasoning are different in different contexts. They may be verbal expressions of embodied principles in Euclidean geometry (such as congruence which embodies the idea of placing one triangle precisely on top of another, or parallel lines where a line is shifted dynamically maintaining corresponding angles and related properties such as those of alternate angles). The principles may be based on observed regularities of arithmetic that are formulated as 'rules' to act as a basis for algebraic proof. Later they may be reformulated once more in terms of set-theoretic definitions of axiomatic systems and reasoning in terms of formal proof.

This framework has been applied to SimCalc by Lima, Healy \& Campos (2012) in this text, interpreting SimCalc as relating real life activities to the mathematical worlds of conceptual embodiment of dynamic graphical representations Reasoning is verbalised and communicated in terms of conceptual embodiment and operational symbolism, but not yet in terms of axiomatic formalism. 
Their analysis includes a significant new reflection on what happens as learners encounter new contexts where their previous experience in terms of ideas that they have 'met before' may be supportive or problematic. Using the terminology of Lima \& Tall (2008) and McGowen \& Tall (2010), supportive 'met-befores' encourage generalization while problematic met-befores impede the learner in making sense of the new situation.

This is coupled with an analysis of emotional reactions using the goal-oriented theory of Skemp (1979), where previous success can increase confidence and encourage students to meet conflict with a determination to overcome difficulties and seek the pleasure of making sense. Alternatively, problematic aspects may lead either to a desire to satisfy external requirements to learn procedures to pass examinations, or worse, to a spiral in which failure leads to avoidance of doing mathematics which in turn leads to more failure and increasing anxiety (Baroody \& Costlick 1998). This link between cognitive success or failure and emotional pleasure or anxiety sheds new light on the nature of the long-term decrease of the number of learners who make sense of mathematics until only a small proportion end up even attempting to succeed in the more sophisticated ideas of the calculus.

It gives a broader view of the whole enterprise of mathematical thinking consonant with Kaput's program for understanding 'Mathematical Change and Variation'. It suggests that technology may be used to give insight into dynamically continuous change through crystallizing the rate of change graphically based on the idea of changing slope built on the notion of local straightness. This may then be expressed symbolically not only in terms of algebraic expressions but more importantly in terms of local linearity which has the potential to develop formally in terms of the traditional definition of limit. It also offers a new vision of the limit concept consistent with the Kaput program which can be constructed from meaningful experiences of conceptual embodiment and operational symbolism.

Modern technology enhances human abilities to make sense of dynamic change through the interactive ability to physically control the variability of motion crystallized as manipulable representations of graphs. It also has the internal capacity to process expressions numerically and symbolically to enable the learner to see the effects of their actions and to share their ideas through human and technological interaction. However, although technology can be used to compute numerically, manipulate symbolically and represent ideas visually, so that it offers the human mind possibilities for future developments, it does not yet have the human capacity to imagine new conceptions and to create new theories. It is therefore important to recognize those aspects that can be supported by technology and those which humans need to develop by using their own mental facilities.

My own personal view is that we need to understand more about how individual human thinkers build mathematical ideas in increasingly sophisticated contexts and how their interpretation of new contexts is affected by fundamentally different emotional reactions to supportive and problematic changes in meaning. In addition we need to consider these changes not only in terms of the children's own learning but also in ourselves as teachers, mathematics educators and theory builders. 


\section{Building on the Confidence of Success}

The transition to new ways of thinking needs to take into account the emotional reactions that feed back into learners' attitudes which can develop cycles of success encouraging more determination to solve new problems or of failure building anxieties that impede future development. This suggests the need to take account of the success of student's thinking processes at one level and to use the confidence that it generates in one situation to be able to realize what is necessary to succeed in new situations.

One possibility in the mathematics of variation and change is to build from the confidence in using piecewise linear physical drawing in SimCalc to shift to the use of a locally straight approach to develop more sophisticated levels of insight in the calculus.

However, the successive changes of meaning - from distances varying in time to the change of distance with respect to time (velocity), then velocity changing in time (acceleration) and acceleration changing in time (jerk) - gives a succession of different meanings that may impede the generality of the mathematics of change. For instance, in the case of simple harmonic motion, the distance is $x=\sin t$, velocity is $\cos t$, acceleration is $-\sin t$ and 'jerk' is $-\cos t$. In what sense can the smooth trigonometric function $-\cos t$ be considered a 'jerk'?

The concept of rate of change itself may be better served by the rate of change of a locally straight graph that may be seen by looking along the graph to see the derivative. If the derivative is again locally straight then the process may be repeated for higher derivatives as long as they are also locally straight.

This extends the Kaput program to local straightness in functions of a single variable. This can be generalized to 'local flatness' of a function of many variables to deal with multi-dimensional calculus, and the same ideas extend to integration, differential equations, partial derivatives, so that the relationship between continuity and local straightness is the foundation of the whole of calculus at all levels (Tall, 1985, 1986, 1989, 2009, 2012).

In the historical vision of the calculus, curves were imagined as polygons with an infinite number of infinitesimal sides. But unlike Kaput, who focused on the finite version of this idea using polygonal curves, I imagined a dynamic version under arbitrary magnification, where I see locally straight curves as looking straight everywhere when highly magnified. Unlike the original vision of such great mathematicians as Barrow, Newton and Leibniz who saw curves with infinitesimal sides and corners that turned through an infinitesimal angle, I encourage today's learners to use technology to see a differentiable function to look straight under high magnification everywhere, with no corners.

This is the resolution of a three and a half century conundrum that is now rationalized in the embodied vision of modern technology. It also happens, by chance, that, going back in history to my own $\mathrm{PhD}$ supervisor, and then to his supervisor, and back to mentors of mentors before PhDs were invented, I am one of 
thousands of mathematicians alive today who can trace his ancestry back 14 generations to Sir Isaac Newton and one further generation to Barrow, who also inspired Leibniz. This is a tenuous link, but it is gratifying to see the vision of the originators of the calculus as a simple dynamic idea that can be democratically shared with the wider population in our technological society.

Building on the dynamic idea of a continuous function that can be drawn with da stroke of a pencil on a sheet of paper, it is simple to translate the embodied idea of continuity to the formal epsilon-delta definition and vice-versa, to show that using a pencil whose point makes a mark covering a square of side length $2 \varepsilon$ it is possible to draw a formally continuous function $f$ from a point $(a, f(a))$ to another point $(b, f(b))$ as a (thick) dynamic pencil line that covers the graph of the theoretical function. (Full details are given in Tall, 2012.)

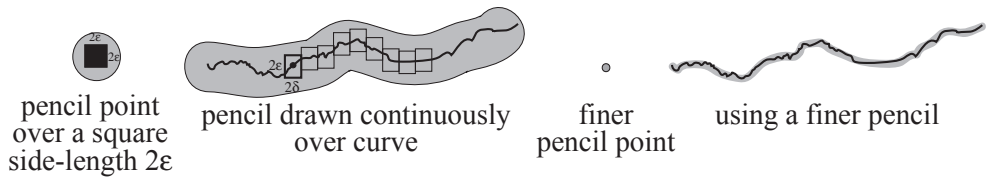

Figure 2. Drawing a continuous graph.

Starting with a continuous function, it may be integrated to give a locally straight area function whose derivative is continuous. Integrating a second time gives a function that is differentiable twice with a second derivative that is continuous. Integrating $n$ times gives a function that can be differentiated $n$ times to give a function that is continuous. If we define a $C^{n}$ function to be a function whose $n$th derivative is continuous, then we see a continuous function as the case $n=0$ at the root of a whole hierarchy of increasingly smooth functions. At the apex we may see $C^{\infty}$ functions that are infinitely differentiable. A simple case is a polynomial that may be differentiated as often as desired and this suggests a generalization from polynomials to power series.

However, as in Kaput's program, we need to develop 'systematic forms of reasoning and argument to help establish the certainty, generality, and reliability of our mathematical assertions.' Contrary to the natural expectation that infinitely differentiable functions are expressible as power series, we find that there are counter-examples. For instance, the function $f(x)=e^{-1 / x^{2}}$ where we take $f(0)=0$ has graph that is so very flat at the origin that all its higher derivatives are zero, so that the power series associated with it is zero while the function itself is not. To be able to cope with such ideas requires a systematic form of reasoning that establishes the reliability of our assertions with clearly defined assumptions. It is for this reason that the calculus requires extension to more formal systematic modes of thinking such as those in mathematical analysis.

While this level of operation is certainly not necessary for the majority of the population, it is essential that we who think about the full range of the mathematics of change and variation encourage teachers in Science, Technology, Engineering and Mathematics (STEM) to have a fundamental grasp of the bigger picture. 


\section{Views of Calculus Appropriate to the Needs of the Individual}

Kaput's view of democracy in terms of making sense of mathematics for the wider community can now be seen in its widest sense, to take account of how individuals play diverse roles in society, each offering his or her own contributions to make the whole so much greater than the sum of its parts. In my new book, How Humans Learn to Think Mathematically (Tall, forthcoming), I study the development of mathematical thinking as individuals mature from new-born children to adults in a wide spectrum of differing ways. This mathematical development builds from perception and action, through the use of symbolism and natural language, to successively more sophisticated forms of mathematical thinking.

In school, mathematics is seen as a blend of what I term conceptual embodiment involving the static and dynamic physical and mental pictures of objects and their properties, and operational symbolism, which begins with actions on objects such as counting, measuring and sharing that are encapsulated into thinkable objects such as whole numbers and generalized as fractions, negatives, decimals, rationals and irrationals, real and complex numbers, and the generalized arithmetic of algebra. For a small minority, there is a development in university to the axiomatic formalism of formal definition and proof.

At every stage there is a divergence in performance in different individuals as some aspects of previous personal experience feature as supportive met-befores in generalizing to a new situation, while other aspects are problematic met-befores that impede conceptual development (Tall, forthcoming, chapter 3).

As human perceptions and operations become more sophisticated through the development of human reasoning, I use van Hiele's (1986) ideas to see mathematics developing broadly through levels of recognition, description, definition and deduction. I see this more as a broad development that encourages children to make sense of mathematics in a meaningful way, rather than performing a microanalysis of various levels to be used in assessment that often provokes teachers to to teach to the test. Broadly speaking, over time, I suggest that three major stages of mathematics occur that I term practical mathematics, theoretical mathematics and formal mathematics (Tall, forthcoming, chapter 1).

Practical mathematics occurs in the geometry of space and shape, through recognition and description of visual and spatial concepts. In arithmetic it occurs through the practical activities of number and measurement, including the recognition and description of properties of arithmetic of whole numbers, fractions, decimals, and negative numbers (which may be introduced in a practical fashion, before or after fractions).

Theoretical mathematics occurs in geometry with the introduction of definitions of figures and their practical constructions using a straight edge and a pair of compasses to draw lines and circles. It continues with the deduction of theorems in Euclidean geometry. In arithmetic, the shift to theoretical mathematics occurs as observed properties of arithmetic are used in the definition of properties of whole 
numbers such as even, odd, prime, composite and the deduction of theorems such as the fact that there is an infinity of primes and that every whole number is expressed uniquely as a product of primes. In algebra, the 'rules of arithmetic' are used as definitions leading to the deduction of various algebraic identities using an algebraic form of proof.

The calculus blends together both embodiment and symbolism in a theoretical approach based on local straightness. It builds on embodiment and symbolism through the perception and recognition of the dynamic changing slope of a graph and the description of the slope function to see the slope functions of $x^{2}, x^{3}, x^{n}$, $\sin x, \cos x$ (with angles in radians), and to look at the graphs of $2^{x}$ and $3^{x}$ to see that they are both increasing graphs with similar slope functions and that as the parameter $a$ in the function $y=a^{x}$ varies between 2 and 3, there may be a numerical value of $e$ between 2 and 3 so that the derivative of $e^{x}$ is again $e^{x}$. By approximating $e^{x}$ as a long polynomial $A+B x+C x^{2}+\ldots$ it is not only possible to find the coefficients but to put $x=1$ to calculate $e$ to, say, ten decimal places by hand or using a simple spreadsheet program. This gives a sense of the idea of a slope function in a range of different examples and also introduces the idea of approximating a function by a power series.

However, to be able to compute the derivatives of composite functions such as $e^{x} \sin \left(x^{2}\right)$, which quickly becomes too complicated to guess by just looking, it becomes necessary to give a more coherent theoretical definition of the limit concept to be able to develop the rules of differentiation to be able to compute derivatives symbolically (Tall, 2012). This definition of a derivative may be formulated in a simply way as the stabilized picture of the practical derivative $(f(x+h)-f(x)) / h$ through the variable point $x$ and for small values of $h$, as $h$ is taken increasingly small.

It is therefore possible to have a theoretical approach to the calculus that does not introduce the concept of limit until it is seen by the learner to be a necessary construct to make sense of computing derivatives.

While the vast majority of the population can make sense of a practical approach to the calculus, as found in SimCalc or in a subsequent development, those who need mathematics in technical applications may be well-served by a theoretical approach to calculus and only the small minority who need to make sense of mathematical analysis may require a formal approach.

The precise nature of practical and theoretical approaches will change as the available technology evolves and affords new ways of making sense of the dynamic notions of continuity and the mathematics of change and variation. SimCalc is a pioneering beginning that has evolved as the technology has evolved. But where will it go in future?

Is it not time for a vision that builds on the evolution of ideas over the millennia and the use of dynamic interactive technology to see and sense the ideas of the local rate of change and of dynamic growth building from human perception and action to the frontiers of mathematical research? 
The framework presented here, as an extension of the Kaput program, encompasses the full range of mathematical development while addressing the wider issues of individual freedom and democracy. It encourages the whole population to gain access to the practical mathematics required for mathematical literacy in a democratic society, for those requiring a more technical approach to build a theoretical form of mathematics of use in applications, and for the pure mathematician to retain any unyielding belief in the necessity of formal mathematics.

It builds on the vision of Jim Kaput to reveal the potential to move into the future, to take advantage of new technologies that give the learner a natural dynamic interface to manipulate enactive imagery and to communicate ideas socially using new technological modes of representation and communication.

\section{Reflections}

Looking back on this chapter in particular and this book as a whole, I have chosen to consider the bigger picture of the Kaput program that is instantiated in the SimCalc program from which a much wider evolution of mathematical change and variation may grow. In using the framework of Kaput to review the practicalities of SimCalc and suggesting new developments for the future, I trust that the reader will not think that I fail to show respect to his memory.

On the contrary, it is the very robustness of the overall Kaput program that enables reflective criticism to encourage us to evolve from current ideas into a future as yet unknown. I affirm that much of my own development has benefited from his profound insights. Indeed, every paper of his that I have read-whether I understood or agreed with everything he said at the time-has contained quotable pearls of wisdom that have profoundly affected my own personal development.

Jim Kaput was the first person to alert me clearly to the active volcano of technology where the mathematical mountain is changing before our eyes. He first made me aware of the relevancy of different symbol systems, though, at the time, I did not fully understand his ideas with any clarity. He was also prescient in the way that he saw even his own insights would need to change as ideas evolve. His profound overall program contains within it the elements for this necessary evolution.

In practice he developed SimCalc software to represent ideas in the mathematics of variability and change and to give democratic access to profound mathematical ideas not expressible within the standard curriculum. His program also extended beyond his remit for working with multiple representations of change and variability, to move on to 'systematic forms of reasoning and argument to help establish the certainty, generality, and reliability of our mathematical assertions.'

It is appropriate at this point to close this chapter, and the whole book, with Jim Kaput's own vision, as follows: 
We see new technologies creating a possibility to reconnect mathematical representations and concepts to directly perceived phenomena, as well as to strengthen students' understanding of connections among different forms of mathematical representation. By starting from more familiar antecedents, such as graphs and motion, both in kinesthetic and cybernetic form, and developing towards more compact and formal mathematical representations, we see an opportunity to create a new path of access to mathematics that has too often remained the province of a narrow elite. (Kaput \& Roschelle, 2012, this volume, p. xxx.)

\section{References}

Baroody, A. J. \& Costlick, R. T. (1998). Fostering children's mathematical power. An investigative approach to $K-8$ mathematics instruction. New Jersey: Lawrence Erlbaum.

Burke, J., Robidoux, R. \& Hegedus, S. (2012). Evolution of MathWorlds. This book pp. xxx$\mathrm{xxx}$

Fleming, D. \& Kaput, J. (1979). Calculus with analytic geometry. New York: Harper \& Row.

Gates, W. (2010). Retrieved from the internet 10 Jan 2012 at http://macdailynews.com/2010/02/ 10/bill_gates_nothing_on_ipad_i_look_at_and_say_oh_i_wish_msft_had_it/

Harel, G. (2000). Three principles of learning and teaching mathematics: Particular reference to linear algebra-Old and new observations. In Jean-Luc Dorier (Ed.), On the Teaching of Linear Algebra, Kluwer Academic Publishers (pp. 177-190).

Harel, G. (2012). The Kaputian Program for the Mathematics of Change and Variation and Its Relation to DNR-Based Instruction in Mathematics. This book, pp. xxx-xxx.

Harel, G., \& Kaput, J. J. (1991). The role of conceptual entities in building advanced mathematical concepts and their symbols. In D. O. Tall (Ed), Advanced Mathematical Thinking. Kluwer Academic Publishers, 82-94.

Hegedus, S. \& Moreno-Armella, L. (2012). Intersecting Representation and Communication Infrastructures. This book, pp. xxx-xxx.

Kaput, J. J. (1992). Technology and Mathematics Education. In D. Grouws (ed.) Handbook on research in mathematics teaching and learning, (pp. 515-556). New York: Macmillan.

Kaput, J. J. \& Roschelle, J. (2012). The Mathematics of Change and Variation from a Millennial Perspective: New Content, New Context. This book, pp. xxx-xxx.

Lima, R. N., Healy, L. \& Campos T. (2012). Adapting SimCalc to Different School Mathematics Cultures. This book, pp. xxx-xxx.

Lima, R. N., \& Tall, D. O. (2008). Procedural embodiment and magic in linear equations. Educational Studies in Mathematics, 67 (1), 3-18.

McGowen, M. C. \& Tall, D. O. (2010). Metaphor or Met-before? The effects of previous experience on the practice and theory of learning mathematics. Journal of Mathematical Behavior $29,169-179$.

Moreno-Armella, L. \& Hegedus, S. J. (2012). From Static to Dynamic Mathematics: Historical and Representational Perspectives. This book, pp. xxx-xxx.

Skemp, R. R. (1979). Intelligence, Learning, and Action. Wiley: London.

Tall, D. O. (1981). Elementary axioms and pictures for infinitesimal calculus, Bulletin of the IMA, 18, 43-48.

Tall, D. O. (1985). Understanding the calculus, Mathematics Teaching, 110, 49-53.

Tall, D. O. (1986). A graphical approach to integration and the fundamental theorem, Mathematics Teaching, 113, 48-51.

Tall, D. O. (1989). Concept Images, Generic Organizers, Computers \& Curriculum Change, For the Learning of Mathematics, 9, 3, 37-42. 
Tall, D. O. (2006). Developing a Theory of Mathematical Growth. International Reviews on Mathematical Education (ZDM), 39, (1-2), 145-154.

Tall, D. O. (2009). Dynamic mathematics and the blending of knowledge structures in the calculus. ZDM - The International Journal on Mathematics Education, 41 (4) 481-492.

Tall, D. O. (2012). A Sensible Approach to the Calculus. To appear in Handbook on Calculus and its Teaching, ed. François Pluvinage \& Armando Cuevas. Available on the internet from http://www.warwick.ac.uk/staff/David.Tall/downloads.html.

Tall, D. O. (forthcoming). How Humans Learn to Think Mathematically. New York: Cambridge University Press.

Tall, D. O., Gray, E. M., Ali, M. B., Crowley, L. F., DeMarois, P., McGowen, M. C., Pitta, D., Pinto, M. M. F., Thomas, M. O. J. \& Yusof, Y. (2001). Symbols and the Bifurcation between Procedural and Conceptual Thinking, Canadian Journal of Science, Mathematics and Technology Education, 1, 81-104.

Van Hiele, P. M. (1986). Structure and Insight. Orlando: Academic Press. 